\title{
Hamiltonian-versus-energy diagrams in soliton theory
}

\author{
Nail Akhmediev and Adrian Ankiewicz \\ Australian Photonics CRC, Optical Sciences Centre, The Australian National University, Canberra 0200, \\ Australian Capital Territory, Australia \\ Roger Grimshaw \\ Department of Mathematics and Statistics, Monash University, Clayton, Victoria 3168, Australia
}

(Received 10 September 1998; revised manuscript received 12 January 1999)

\begin{abstract}
Parametric curves featuring Hamiltonian versus energy are useful in the theory of solitons in conservative nonintegrable systems with local nonlinearities. These curves can be constructed in various ways. We show here that it is possible to find the Hamiltonian $(H)$ and energy $(Q)$ for solitons of non-Kerr-law media with local nonlinearities without specific knowledge of the functional form of the soliton itself. More importantly, we show that the stability criterion for solitons can be formulated in terms of $H$ and $Q$ only. This allows us to derive all the essential properties of solitons based only on the concavity of the curve $H$ vs $Q$. We give examples of these curves for various nonlinearity laws and show that they confirm the general principle. We also show that solitons of an unstable branch can transform into solitons of a stable branch by emitting small amplitude waves. As a result, we show that simple dynamics like the transformation of a soliton of an unstable branch into a soliton of a stable branch can also be predicted from the $H$ - $Q$ diagram. [S1063-651X(99)09805-0]
\end{abstract}

PACS number(s): 42.65.-k, 47.20.Ky, 47.27.Te

\section{INTRODUCTION}

The Hamiltonian $(H)$ is one of the fundamental notions in mechanics [1] and more generally in the theory of conservative dynamical systems with a finite (or even infinite) number of degrees of freedom. The Hamiltonian formalism has turned out to be one of the most universal in the theory of integrable systems [3] and nonlinear waves in general [2]. In the case of nonintegrable systems, the Hamiltonian exists whenever the system is conservative, and it is useful for stability analysis [4,5]. It turns out that the most useful approach in soliton theory of conservative nonintegrable Hamiltonian systems is a representation on the plane of conserved quantities: Hamiltonian versus energy [6]. A threedimensional (3D) plot (Hamiltonian-energy-momentum) is useful when dealing with two-parameter families of solutions [7].

Recently, Hamiltonian-versus-energy curves have been used effectively to study families of solitons and their properties, viz., range of existence, stability, and general dynamics. Specific problems considered up to now include scalar solitons in non-Kerr media [6], vector solitons in birefringent waveguides [8], radiation phenomena from unstable soliton branches [9], optical couplers [10], general principles of coupled nonlinear Schrödinger equations [11,12], parametric solitons in quadratic media [13], and the theory of BoseEinstein condensates [14]. Moreover, Hamiltonian-versusenergy curves are useful not only for studying single-soliton solutions, but also for analyzing the stability of bound states (when they exist) [15]. Other examples could be mentioned as well.

In most publications, soliton families have been studied using plots of energy versus propagation constant. These curves allow the soliton families to be presented graphically and, moreover, allow predictions of their stability properties.
We believe that the first example of their application was presented in [16]. Kusmartsev [17] was the first person to understand the importance of projecting curves on the plane of conserved quantities. He applied catastrophe theory and a mapping technique to represent soliton families with diagrams and to show that the critical points on these diagrams define the bifurcations where the soliton stability changes. However, the qualitative analysis in the work has been simplified and it missed some important details. In particular, for infinite-dimensional systems, the parametrization of wave packets using two parameters ( $\omega$ and $k$ in [17]) is valid only in the close vicinity of stationary solutions where the Hamiltonian has an extremum. For more general solutions and for the evolution of a wave packet from an arbitrary initial condition, the use of the above parameters may fail. On the other hand, at the extremal points, more definite parametrization is needed.

In this work, we use a direct approach to analyze the $H(Q)$ soliton curves and, additionally, we enhance the concept with a stability theorem. We believe that this theorem turns the employment of $H(Q)$ curves into a powerful tool for analyzing soliton solutions, their stability, and their dynamics.

Usually the Hamiltonian and energy for solitons of nonKerr media are found by substituting the explicit soliton form into the appropriate integrals. However, the explicit forms are not always available, and furthermore, not always necessary. Sometimes, it is sufficient to know that the fundamental soliton can be represented as a single-peak function which decreases to zero at infinity. Then the important properties of solitons-range of existence, stability, and simple dynamics - can be predicted from our analysis. In particular, in this paper, we prove a theorem which relates the concavity of the $H-Q$ curves to the stability of the solitons. We consider several examples of local nonlinearities and apply the 
general principles to these media. The main advantages of our approach are its simplicity, clarity, and the fact that it provides the possibility of predicting simple dynamics of evolution for solitons on unstable branches.

\section{ANALYSIS}

For simplicity, we consider in this paper only scalar wave fields $\psi(t, \xi)$. The nonlinear Schrödinger equation (NLSE) for a general nonlinearity law is [4-6]

$$
i \psi_{\xi}+\frac{1}{2} \psi_{t t}+N\left(|\psi|^{2}\right) \psi=0
$$

In the case of temporal solitons, $t$ is the retarded time variable [while in the case of spatial $(1+1) \mathrm{D}$ solitons, $t$ is a transverse spatial coordinate], $\xi$ is the longitudinal distance, and $N$ is the nonlinearity law. It indicates that the change in refractive index depends on the local intensity. Localized solutions satisfy the ansatz

$$
\psi(t, \xi)=f(t) \exp (i q \xi)
$$

where $f(t)$ is a real field profile, and $q$ is the propagation constant.

The total energy associated with an arbitrary solution, $\psi(t, \xi)$, is

$$
Q=\int_{-\infty}^{\infty} I d t
$$

where the intensity is $I=|\psi|^{2}=f^{2}$. Strictly speaking, in spatial problems, $Q$ is the power or power flow. In problems related to pulse propagation in optical fibers, where $t$ is regarded as a retarded time, $Q$ is the total pulse energy. For simplicity, we refer to $Q$ as energy throughout this work, keeping in mind the above remark. For localized solutions [Eq. (2)], $Q$ is finite and it is one of the conserved quantities of Eq. (1).

Similarly, the Hamiltonian is another conserved quantity:

$$
H=\int_{-\infty}^{\infty}\left[\frac{1}{2} f_{t}^{2}-F(I)\right] d t,
$$

with $F$ given by

$$
F(I)=\int_{0}^{I} N\left(I^{\prime}\right) d I^{\prime}
$$

The Hamiltonian plays a major role in the dynamics of the infinite-dimensional system. Namely, stationary solutions of Eq. (1) can be derived from the Hamiltonian using the variational principle $\delta H=0$.

Now, substituting Eq. (2) into Eq. (1) and integrating once, we have

$$
f_{t}^{2}=2(q I-F)
$$

Using Eqs. (4) and (5), it is easy to show that

$$
H=q Q-2 K
$$

where $K=\int_{-\infty}^{\infty} F(I) d t$. The point now is that we can convert from integrals in the time domain $(-\infty<t<\infty)$ to integrals in terms of the intensity $\left(0<I<I_{m}\right.$ where $I_{m}$ is the maximum pulse intensity). We suppose that the soliton profile is a single maximum solution of Eq. (1) with no nodes. In other words, we deal with the fundamental nonlinear "mode" of Eq. (1). We then do not need to solve the modified NLSE explicitly. We use the fact that

$$
\frac{d I}{d t}=2 f f_{t}=-2 \sqrt{I} \sqrt{2(q I-F)}
$$

$(t>0)$ to obtain

$$
Q \sqrt{2 q}=\int_{0}^{I_{m}} \frac{d I}{\sqrt{1-J(I)}}=I_{m} \int_{0}^{1} \frac{d y}{\sqrt{1-J\left(I_{m} y\right)}},
$$

where we have defined $J(I)=F(I) / q I$ and introduced the change of variable $y=I / I_{m}$ for convenience. We note that $J\left(I_{m}\right)=1$, so that the condition $J(I) \leqslant 1$ determines the existence regime of soliton solutions. Furthermore,

$$
K \sqrt{\frac{2}{q}}=\int_{0}^{I_{m}} \frac{J(I) d I}{\sqrt{1-J(I)}} .
$$

Then $Q q-K$ simplifies to $S / \sqrt{2}$, where

$$
S=\sqrt{q} \int_{0}^{I_{m}} \sqrt{1-J(I)} d I=I_{m} \sqrt{q} \int_{0}^{1} \sqrt{1-J\left(I_{m} y\right)} d y .
$$

Finally

$$
H=\sqrt{2} S-q Q
$$

This expression will be used here to calculate $H$-versus- $Q$ curves explicitly. It is easy to show that in the case of a Kerr medium

$$
H(Q)=-\frac{Q^{3}}{24}
$$

\section{STABILITY THEOREM}

One of the advantages of using $H-Q$ curves is that they can predict the stability of solitons. It is apparent that, if there is more than one branch at a given $Q$, then the lowest branch (i.e., the one with the minimum Hamiltonian) is stable. This conclusion follows directly from the nature of the Hamiltonian and does not need a special proof. However, we will show that the stability condition can take a more direct form. We now prove a useful theorem in this regard. For solitons in media with local nonlinearities, we have, using the equations in Sec. II,

$$
\frac{d S}{d q}=\frac{1}{2 q}(S+K \sqrt{2}) .
$$




$$
\frac{d H}{d q}=-q \frac{d Q}{d q} .
$$

Then it follows that

$$
\frac{d H}{d Q}=-q
$$

If we start at $q=0$ and traverse the curve so that $q$ is increasing, then the magnitude of the slope always increases. Furthermore,

$$
\frac{d^{2} H}{d Q^{2}}=\frac{-1}{d Q / d q}
$$

The denominator on the right-hand side defines the stability of the lowest-order modes (fundamental solitons) [4,19-23]. Now we can see that stability is directly related to the concavity of the $H$-versus- $Q$ curve. Namely, the solitons with $H^{\prime \prime}(Q)<0$ are stable while those with $H^{\prime \prime}(Q)>0$ are unstable.

Another consequence of Eq. (14) is that

$$
\frac{d H}{d q}=0 \Rightarrow \frac{d Q}{d q}=0 \quad \text { or } \quad q=0 .
$$

Thus if $Q$ has a stationary point, then so does $H$. For $q>0$, this produces a cusp on the $H-Q$ diagram. However, we can have $d H / d q=0$ with $q=0$ and $d Q / d q \neq 0$. This produces a rounded maximum on the $H$-vs- $Q$ plot and not a cusp.

Clearly, from Eq. (14), if we have $q>0$, then $H$ decreases as $Q$ increases, meaning that $d H / d Q<0$. On the other hand, if $q<0$ is allowable, then $H$ and $Q$ have the same slope, so that $d H / d Q>0$.

Thus, we can conclude the following, for the lowest-order modes.

(1) Solitons with $H^{\prime \prime}(Q)<0$ are stable while those with $H^{\prime \prime}(Q)>0$ are unstable.

(2) Stability changes only at cusps.

This criterion for stability can be more general than $d Q / d q>0$, because it involves only conserved quantities which always exist in conservative systems; this is in contrast to $q$, which may not be defined uniquely. This is an important theorem and we illustrate its application in several of the following examples. Moreover, we also consider what happens to unstable solitons if they are excited in the system.

\section{EXAMPLES}

\section{A. Power-law nonlinearity}

This nonlinearity has been studied in relation to the selffocusing singularity [18]. Here $N=I^{b}$, with $b>0$, so that $J$ $=\left(I / I_{m}\right)^{b}$. Usually, we have $b<2$ so that the solitons do not collapse (in the one-dimensional case). Then

$$
S=\frac{b Q \sqrt{2} q}{b+2},
$$

and

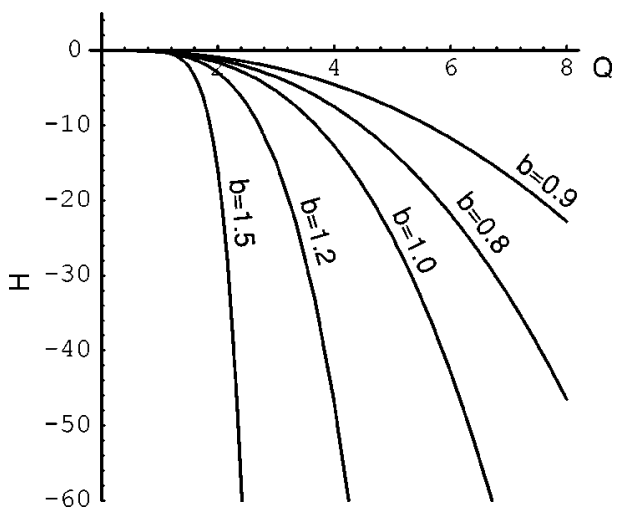

FIG. 1. Hamiltonian versus energy for power-law nonlinearity for various values of the parameter $b$.

$$
H(q, Q)=\frac{b-2}{b+2} q Q .
$$

We define

$$
c(b)=\left[\sqrt{\frac{2}{\pi}} \frac{b \Gamma(1 / b+1 / 2)}{(1+b)^{1 / b} \Gamma(1 / b)}\right]^{2 b /(2-b)},
$$

where $\Gamma$ is the gamma function. Then $Q$ is given by

$$
Q=\left[\frac{q}{c(b)}\right]^{(2-b) / 2 b}
$$

and

$$
H(Q)=c(b) \frac{b-2}{b+2} Q^{(2+b) /(2-b)} .
$$

Thus, the $H$-versus- $Q$ curve can be calculated without any knowledge of the soliton profile itself. The curves $H(Q)$ are shown in Fig. 1 for several values of the parameter $b$. When $b=1$ (Kerr medium), then $c(b)=1 / 8$ and $H(Q)=-Q^{3} / 24$, in agreement with Eq. (12).

Let us consider stability. The derivative,

$$
\frac{d H}{d Q}=-c(b) Q^{2 b /(2-b)}=-q
$$

as required, and

$$
\frac{d^{2} H}{d Q^{2}}=-\frac{2 b c(b)}{(2-b)} Q^{(3 b-2) /(2-b)}=-\frac{1}{Q^{\prime}(q)}
$$

Solitons in these media are always stable. Note that when $0<b<2$, these functions are single valued, so that all solitons of the family are stable and $H^{\prime \prime}(Q)$ is always negative. Thus the curve is always concave down. The latter fact is important when considering inelastic interactions between solitons [15].

Clearly, $b=2$ represents the borderline case between the concave downwards curves $(b<2)$ and the concave upwards curves $(b>2)$. In fact, for $b=2$ the curves reduce to a single point $(Q=\sqrt{3 / 2} \pi / 2 \approx 1.924, H=0)$, and this is independent of $q$. In this case, the exponential growth rate coefficient 
is zero and the stability (linear growth) would have to be considered separately, as there is no concept of concavity for a single point.

Although the actual field profile has not been used, for the sake of completeness, we give it here:

$$
\left.f(t)=[q(1+b)]^{1 / 2 b} \operatorname{sech}^{1 / b}(b \sqrt{2 q} t)\right] .
$$

If we set $b=1$, then we obtain

$$
f(t)=\sqrt{2 q} \operatorname{sech}(\sqrt{2 q} t),
$$

which is the well-known Kerr-law soliton.

\section{B. Log-law nonlinearity}

Nonlinearity models involving logarithm-type laws allow us to find a multiplicity of exact solutions of Eq. (1) $[24,25]$. Nevertheless, these models remain generally nonintegrable in the sense that the inverse scattering technique cannot be applied to them. In this case, we choose the model $N$ $=\ln \left(b^{2 a} I^{a}\right)=a \ln \left(b^{2} I\right)$, where $a>0$, so that $I_{m}=b^{-2} \exp (1$ $+q / a)$. Then

$$
S=\frac{a Q}{\sqrt{2}},
$$

and

$$
H(q, Q)=(a-q) Q,
$$

while $Q$ is $Q=I_{m} \sqrt{\pi /(2 a)}$, which is proportional to $\exp (q / a)$. Thus

$$
H(Q)=a Q\left[2-\ln \left(b^{2} Q \sqrt{\frac{2 a}{\pi}}\right)\right] .
$$

The effect described in Sec. III shows up clearly with this log-law example. Here

$$
\frac{d H}{d q}=-q \frac{d Q}{d q}=-\frac{q}{a b^{2}} \sqrt{\frac{\pi}{2 a}} \exp \left(1+\frac{q}{a}\right) .
$$

In this case, $H$ has a maximum when $q=0$, and $d Q / d q$ is not zero at this parameter value. Hence, the $H-Q$ plot features a rounded maximum (and not a cusp) at this point, as is clear from Fig. 2.

As seen in the figure, $d H / d Q>0$ for all values of $Q$ below the maximum in $H$ (i.e., $-\infty<q<0$ ), while $d H / d Q$ $<0$ for all values of $Q$ above the maximum in $H$ (i.e., $q$ $>0$ ). Thus no cusps appear in this example.

The slope of the curve of Eq. (28) is

$$
\frac{d H}{d Q}=a\left[1-\ln \left(b^{2} Q \sqrt{2 a / \pi}\right)\right]=-q
$$

so that $H^{\prime \prime}(Q)=-1 / Q^{\prime}(q)=-a / Q$, which is always negative. Some examples are shown in Fig. 2. Note that the Hamiltonian for these curves increases at low energies before decreasing at high energies. These curves are always concave down for any $a$, so the solitons are always stable.

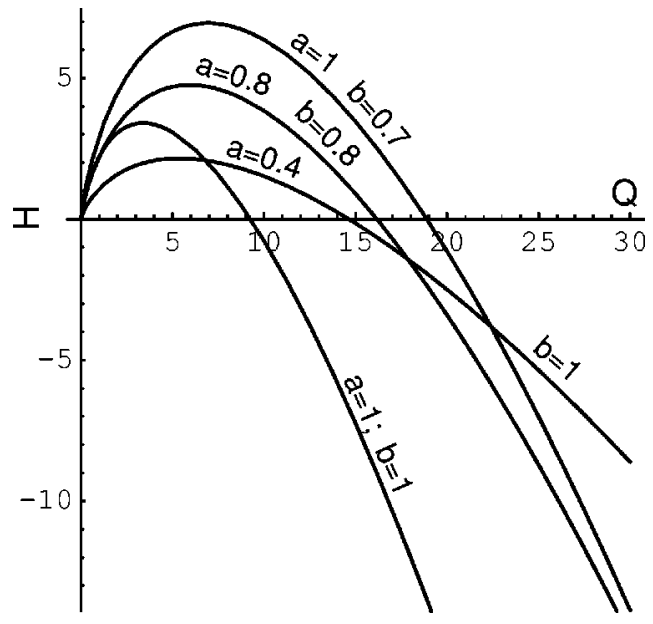

FIG. 2. Hamiltonian versus energy for the logarithmic-law nonlinearity model of this section for various values of the parameters $a$ and $b$.

\section{Cubic-quintic nonlinearity law}

For an arbitrary function $N(I)$, the first two terms in the Taylor series give $N=I+\nu I^{2}$. This nonlinearity can be obtained by using two separate dopants [26]. This model gives $J(I)=(I / q)\left(\frac{1}{2}+\nu I / 3\right)$. Let us consider separately the two opposite signs of $\nu$.

\section{1. $\nu$ positive case}

Now $\nu>0$, so it is convenient to define $\alpha=4 \sqrt{\nu q / 3}$ $\equiv \tan (A)(>0)$. Thus $0<\nu q<\infty$. Then

$$
\begin{aligned}
Q & =\frac{3(\sec A-1)}{4 \nu \sqrt{2 q}} \int_{0}^{1} \frac{d y}{\sqrt{1-y} \sqrt{1+y \tan ^{2}(A / 2)}} \\
& =\sqrt{\frac{3}{2 \nu}} \arctan (\alpha) .
\end{aligned}
$$

Now, using Eq. (10) we find

$$
S=\frac{3 \sqrt{q}}{4 \nu}(\sec A-1) \int_{0}^{1} \sqrt{1-y} \sqrt{1+y \tan ^{2}(A / 2)} d y,
$$

so that

$$
S=\frac{3 \sqrt{3}}{32 \nu^{3 / 2}}\left[\left(1+\alpha^{2}\right) \arctan (\alpha)-\alpha\right] .
$$

Finally, using Eq. (11) we obtain

$$
H(q)=\frac{3 \sqrt{6}}{32 \nu^{3 / 2}}[\arctan (\alpha)-\alpha] .
$$

So, using Eq. (14), we find

$$
\frac{d H}{d q}=-q \frac{d Q}{d q}=-\frac{\sqrt{2 q}}{1+\alpha^{2}} .
$$


Thus, if $q>0$, the slopes $H^{\prime}(q)$ and $Q^{\prime}(q)$ have opposite signs, so that if either $Q$ or $H$ is increasing then the other is decreasing.

We can now give $H$ explicitly in terms of $Q$ :

$$
H(Q)=\frac{3 Q}{16 \nu}-\frac{3 \sqrt{6}}{32 \nu^{3 / 2}} \tan \left(Q \sqrt{\frac{2 \nu}{3}}\right) .
$$

Differentiating shows that

$$
\frac{d H}{d Q}=-\frac{3}{16 \nu} \tan ^{2}(Q \sqrt{2 \nu / 3})=-q
$$

and

$$
\frac{d^{2} H}{d Q^{2}}=-\left(1+\alpha^{2}\right) \sqrt{q / 2}=\frac{-1}{Q^{\prime}(q)} .
$$

\section{2. $\nu$ negative case}

Here $\nu<0$, so we let $\beta=4 \sqrt{-\nu q / 3} \equiv \tanh (B)(>0)$. Thus $0<\beta<1$, so that solitons can exist (only) within the range $-\frac{3}{16}<\nu q<0$. Then

$$
\begin{aligned}
Q & =\frac{3(\operatorname{sech} B-1)}{4 \nu \sqrt{2 q}} \int_{0}^{1} \frac{d y}{\sqrt{1-y} \sqrt{1-y \tanh ^{2}(B / 2)}} \\
& =\sqrt{\frac{-3}{2 \nu}} \operatorname{arctanh}(\beta) .
\end{aligned}
$$

The energy results [Eqs. (31) and (37)] agree with those calculated using the field solutions in Sec. 4.5 of [6].

Now, again using Eq. (10) we get

$$
S=\frac{3 \sqrt{q}}{4 \nu}(\operatorname{sech} B-1) \int_{0}^{1} \sqrt{1-y} \sqrt{1-y \tanh ^{2}(B / 2)} d y,
$$

so that this time $S$ is given by

$$
S=\frac{3 \sqrt{3}}{32 \nu \sqrt{-\nu}}\left[\left(1-\beta^{2}\right) \operatorname{arctanh}(\beta)-\beta\right] .
$$

From Eq. (11) we get

$$
\begin{gathered}
H(q)=\frac{3 \sqrt{6}}{32 \nu \sqrt{-\nu}}[\operatorname{arctanh}(\beta)-\beta], \\
H(Q)=\frac{3 Q}{16 \nu}+\frac{3 \sqrt{6}}{32(-\nu)^{3 / 2}} \tanh \left(Q \sqrt{\frac{-2 \nu}{3}}\right) .
\end{gathered}
$$

We note that Eq. (41) agrees with Eq. (4.30) in [6], with the latter being derived in quite a different manner. Taking the limit $\nu \rightarrow 0$ in Eqs. (36) and (41) again produces the correct Kerr-law limit, $H=-Q^{3} / 24$.

Curves plotted from Eqs. (36) and (41) are shown in Fig. 3. When $\nu$ is positive, each curve has a maximum possible $Q$. When $\nu$ is negative, there are no limits along either variable. In both cases, $H^{\prime \prime}(Q)<0$, so all these solitons are stable.

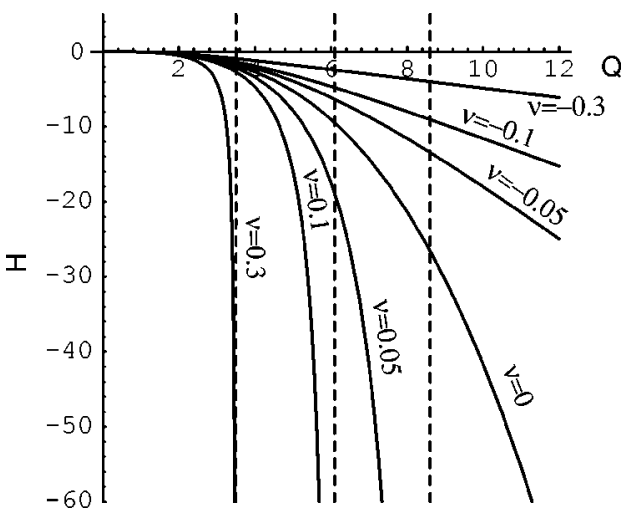

FIG. 3. Hamiltonian versus energy for cubic-quintic nonlinearity for various values of the parameter $\nu$.

\section{Higher-order polynomial law}

A more general case of a Taylor expansion has been considered by Kaplan [27]. Here, we can, to some extent, generalize the preceding section by using $N=I+\nu I^{2}+\gamma I^{3}$. Thus

$$
J(I)=\frac{I}{q}\left(\frac{1}{2}+\frac{\nu I}{3}+\frac{\gamma I^{2}}{4}\right) .
$$

Here $I_{m}$ is the first (positive) root of $3 \gamma I_{m}^{3}+4 \nu I_{m}^{2}+6 I_{m}$ $-12 q$. This can be written in an explicit form.

Now $1-J\left(I_{m} y\right)$ is a cubic polynomial in $y$ and it has $y$ $=1$ as a root. Hence $1-J\left(I_{m} y\right)=(1-y)\left(1+a y+b y^{2}\right)$. Expanding shows that $a=1-I_{m} / 2 q$ and $b=\gamma I_{m}^{3} / 4 q$. Then we find

$$
Q=\frac{I_{m}}{\sqrt{2 q}} \int_{0}^{1} \frac{d y}{\sqrt{1-y} \sqrt{1+a y+b y^{2}}},
$$

which can be expressed in terms of an elliptic $F$ function, and

$$
S=I_{m} \sqrt{q} \int_{0}^{1} \sqrt{1-y} \sqrt{1+a y+b y^{2}} d y,
$$

which can be written in terms of elliptic $F$ and $\Pi$ functions.

We can make a simplification by writing $1+a y+b y^{2}$ $=\left(1-r_{1} y\right)\left(1-r_{2} y\right)$ where $r_{1}, r_{2}=\frac{1}{2}\left[-a \pm \sqrt{a^{2}-4 b}\right]$. Of course, $r_{1}$ and $r_{2}$ may be complex, but if they are real they cannot be greater than 1 . Then

$$
Q=\frac{I_{m}}{\sqrt{1-r_{2}}} \sqrt{\frac{2}{q r_{1}}} F\left(\arcsin \left(\sqrt{r_{1}}\right), \frac{r_{2}-r_{1}}{r_{1}\left(r_{2}-1\right)}\right) .
$$

In principle, this result may give bistable behavior of solitons [27].

\section{E. Saturable nonlinearity law}

Here we use a nonlinearity model which has been considered in [28], viz.,

$$
N=k\left[1-(1+I / \gamma)^{-2}\right],
$$

where $\gamma$ is the saturation parameter and $k$ is a constant. This nonlinearity does not allow explicit solutions for $f(t)$. How- 


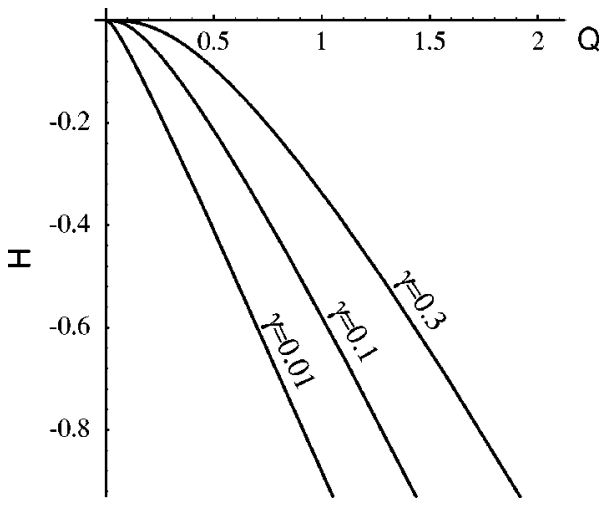

FIG. 4. Hamiltonian versus energy for saturable nonlinearity for three values of the parameter $\gamma$.

ever, $H(Q)$ curves can be calculated analytically using the above formalism. We obtain simple results with our approach, as

$$
F=\frac{I^{2} k}{I+\gamma}, \quad J(I)=\frac{I k}{q(I+\gamma)}, \quad \text { and } \quad I_{m}=\frac{q \gamma}{k-q} .
$$

Then the integrals can be calculated analytically:

$$
Q=\frac{\gamma k}{2 \sqrt{2}(k-q)^{3 / 2}}\left[\frac{\pi}{2}+\frac{2}{k} \sqrt{q} \sqrt{k-q}-C\right]
$$

and

$$
S=\gamma\left[\frac{k}{4 \sqrt{k-q}}(\pi-2 C)-\sqrt{q}\right]
$$

where

$$
C=\arctan \left[\frac{k-2 q}{2 \sqrt{q} \sqrt{k-q}}\right] .
$$

Then $H$ is found from Eq. (11), as before.

In general, $Q$ increases with $q$ while $H$ decreases with $q$. Parametric plots (for $0<q<k$ ) are shown in Fig. 4. They decrease monotonically for any positive $\gamma$ and are concave down, implying stability.

\section{F. Dual power-law nonlinearity}

This nonlinearity is given by $N=I^{b}+\nu I^{2 b}$. When $\nu$ is positive, the refractive index increases monotonically with $I$. Qualitative behavior of $H(Q)$ curves is then similar to the one considered in the preceding section. We consider $\nu<0$, where the $N(I)$ dependence is not monotonic and we can expect qualitatively new effects. We let $\beta=2(1$ $+b) \sqrt{-\nu q /(2 b+1)} \equiv \tanh (B)(>0)$. Thus $0<\beta<1$, so that solitons can exist (only) within the range

$$
-\frac{1+2 b}{4(1+b)^{2}}<\nu q<0 \text {. }
$$

Then

$$
Q=\frac{I_{m}}{b \sqrt{2 q}} \int_{0}^{1} \frac{y^{1 / b-1} d y}{\sqrt{1-y} \sqrt{1-y \tanh ^{2}(B / 2)}},
$$

where

$$
I_{m}=\left[\frac{(1+2 b)[\operatorname{sech}(B)-1]}{2 \nu(1+b)}\right]^{1 / b}
$$

so that

$$
Q=\frac{I_{m}}{b} \sqrt{\frac{\pi}{2 q}} \frac{\Gamma(1 / b)}{\Gamma\left(\frac{1}{2}+1 / b\right)} F\left(\frac{1}{2}, \frac{1}{b} ; \frac{1}{2}+\frac{1}{b} ; z\right),
$$

where $F$ is the hypergeometric function and $z=\tanh ^{2}(B / 2)$. With the special case $b=1, Q$ reduces to

$$
Q=I_{m} \sqrt{\frac{2}{q}} F\left(\frac{1}{2}, 1 ; \frac{3}{2} ; z\right)=I_{m} \sqrt{\frac{2}{q z}} \operatorname{arctanh}(\sqrt{z})
$$

which reduces to the form of $Q$ given in the earlier section by Eq. (37).

Now, again using Eq. (10), we get

$$
S=\frac{I_{m} \sqrt{q}}{b} \int_{0}^{1} y^{1 / b-1} \sqrt{1-y} \sqrt{1-y \tanh ^{2}(B / 2)} d y,
$$

so that $S$ is given by

$$
S=\frac{I_{m} \sqrt{q \pi}}{2 b} \frac{\Gamma(1 / b)}{\Gamma\left(\frac{3}{2}+1 / b\right)} F\left(-\frac{1}{2}, \frac{1}{b} ; \frac{3}{2}+\frac{1}{b} ; z\right) .
$$

When $b=1, S$ agrees with Eq. (39). When $b=2$, the hypergeometric functions reduce to elliptic integrals ( $K$ and $E$ ).

Now we have explicit forms for $Q, S$, and hence $H$. In general, when $b<2, Q$ increases and $H$ decreases monotonically with $q$, so that the parametric $H$ versus $Q$ plot decreases monotonically as $q$ increases and is always concave down. Hence, the solitons of the whole family are stable.

For $b>2$, however, $Q$ has a minimum and $H$ has a maximum at $q>0$, thus producing a cusp in the $H$-versus- $Q$ plot (see Fig. 5). Note that solitons exist only above some threshold energy in this case. The important conclusion from this case is that the upper branch should be unstable, because the Hamiltonian is concave upwards while the lower branch should be stable as it is concave downwards. Numerical simulations similar to that described in Sec. V show that this is indeed the case.

\section{G. Triple power-law extension}

We can also take $N=I^{b}+\nu I^{2 b}+\gamma I^{3 b}$. If we let $n=I_{m}^{b}$, then we can find $n$, and hence $I_{m}$, by solving the cubic equation

$$
\frac{n}{b+1}+\frac{\nu}{2 b+1} n^{2}+\frac{\gamma}{3 b+1} n^{3}=q
$$




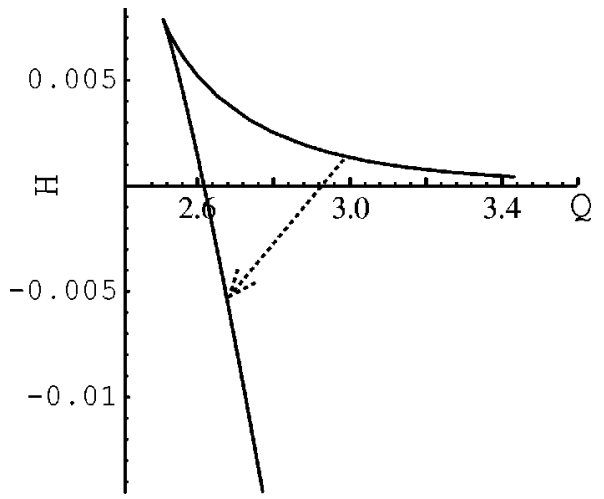

FIG. 5. Hamiltonian versus energy for dual-power-law nonlinearity for the values of the parameters $b=5 / 2$ and $\nu=-1$. The dotted arrow shows a transformation which occurs from the unstable branch to the stable one, due to the soliton's interaction with radiation. The cusp occurs at $q=q_{c}=0.0492$ and corresponds to the soliton's minimum energy of $Q=2.51$ and maximum Hamiltonian, viz., $H=0.00783$.

$$
Q=\frac{I_{m}}{b \sqrt{2 q}} \int_{0}^{1} \frac{y^{1 / b-1} d y}{\sqrt{1-y} \sqrt{1-r_{1} y} \sqrt{1-r_{2} y}},
$$

where the roots of the cubic polynomial are $1,1 / r_{1}$, and $1 / r_{2}$ as before. Again, if $r_{1}$ and $r_{2}$ are real then each must be less than 1.

Similarly

$$
S=\frac{I_{m} \sqrt{q}}{b} \int_{0}^{1} y^{1 / b-1} \sqrt{1-y} \sqrt{1-r_{1} y} \sqrt{1-r_{2} y} d y .
$$

This may put an additional branch on the $H-Q$ curve, leading to bistable behavior.

As noted in Sec. III, the magnitude of the slope of the curve on the $(H-Q)$ diagram increases as we move along the curve in the direction of increasing $q$. For example, in Fig. 6(a), $q=0$ corresponds to the origin, and the magnitude $|d H / d Q|$ increases as we move towards the cusp; this value continues to increase once we move onto the upper branch ( $q>0.25$ ) where $H$ is increasing.

In Fig. 6(b) small $q$ corresponds to the high $Q$ values on the right of the diagram. Again, $|d H / d Q|$ increases as $q$ increases and we approach the first cusp, which is the left cusp. Then $|d H / d Q|$ increases again with $q$ as we move along the lowest branch (i.e., the stable one). When we pass the right cusp, $H$ increases and $|d H / d Q|$ once again continues to increase with $q$.

\section{SOLITON TRANSFORMATION}

The main point of the above calculations is that the exact solution for the soliton profile is not needed for finding the $H(Q)$ curve. Indeed, the explicit forms of solutions $f(t)$ have not been used in these calculations. Nevertheless, we now present an explicit solution for the profile $f(t)$, for the case of dual-power-law solitons in order to investigate the dynamics and verify the usefulness of the $H-Q$ diagram in predicting stability (instability) and pulse behavior.

Thus, for $N=I^{b}+\nu I^{2 b}$ (Sec. IV F), we have the ordinary differential equation:
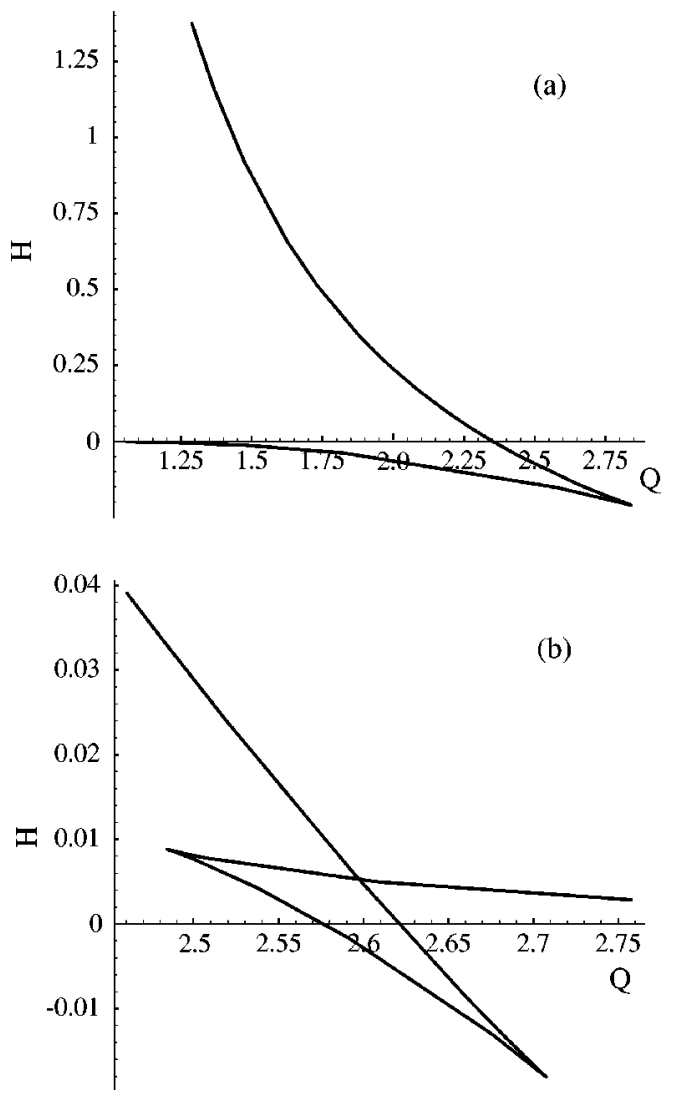

FIG. 6. Hamiltonian versus energy for triple-power-law nonlinearity (Sec. IV G) for the values of the parameters $\nu=-1$ and $\gamma$ $=0.5$. (a) $b=3 / 2$. Here the lower branch is stable and the upper branch is unstable. (b) $b=5 / 2$. Here the lowest branch $(0.06<q$ $<0.17)$ is stable and two upper branches are unstable. Note $d H / d Q=-q$ at each point.

$$
\frac{1}{2} f_{t t}-q f+f\left(f^{2 b}+\nu f^{4 b}\right)=0
$$

The exact solution is

$$
f(t)=[h(t)]^{-1 / 2 b},
$$

where

$$
h(t)=\frac{1}{2 q(1+b)}[1+s(b) \cosh (2 b \sqrt{2 q} t)],
$$

with $s(b)$ defined as

$$
s(b)=\sqrt{1+\frac{4 q \nu}{(1+2 b)}(1+b)^{2}}(>0) .
$$

Using the definitions of previous sections, we note that if $\nu$ $<0$, then $s=\sqrt{1-\beta^{2}}=\operatorname{sech}(B)(<1)$, while if $\nu>0$, then $s=\sqrt{1+\alpha^{2}}=\sec (A)(>1)$. Using Eq. (60), we see that

$$
I_{m}=[f(0)]^{2}=\left[\frac{(1+2 b)(s(b)-1)}{2 \nu(1+b)}\right]^{1 / b} .
$$

For $\nu<0$, this clearly agrees with the form found [Eq. (51)] in the preceding section. 

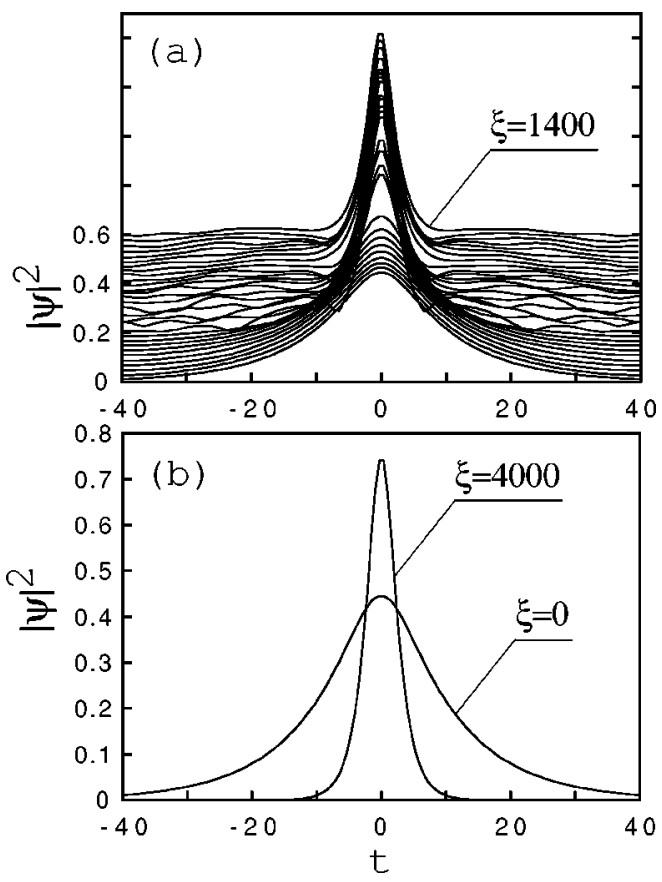

FIG. 7. (a) Evolution of an unstable soliton. The result of this evolution is shown schematically by the arrow in Fig. 5. (b) Initial $(\xi=0)$ and final $(\xi>1400)$ soliton profiles. Initially the stationary soliton solution $(q=0.005)$ is unstable, but it evolves into a soliton on the stable branch while emitting small amplitude radiation waves [note ripples in (a)].

To illustrate the usefulness of the $H(Q)$ diagrams in predicting dynamics, let us consider a simple example. In Fig. 5, the upper unstable branch of solitons corresponds to the range $0<q<q_{c}$. The lower stable branch corresponds to the interval $q_{c}<q<q_{\max }=6 / 49$. The cusp appears at $q=q_{c}$ $=0.0492$. We have made numerical simulations based on Eq. (1) with the initial conditions corresponding to the stationary solutions of the unstable branch. We used the CrankNicholson technique, in conjunction with a Newton iteration scheme, to solve the nonlinear equation. We used zero boundary conditions and absorbing layers close to the boundary in order to remove the small amplitude radiation waves. An example of propagation is shown in Fig. 7(a). These simulations confirm the instability of the upper branch. We start with the exact solution, Eq. (60), as the initial condition, and take $q=0.005$, which corresponds to $Q=2.936$. Initial symmetric perturbations are inserted by multiplying the function by a coefficient slightly different from one, namely, 1.00001 . The results were qualitatively the same even without this coefficient, due to the unavoidable deviations of the profile from the exact one in the numerical discretization. This soliton is unstable, and due to interaction with radiation, it evolves into a soliton of the stable branch. The initial and the final soliton profiles are shown in Fig. 7. The final state, after the radiation waves have dispersed, is a soliton with parameters $q=0.094$ and $Q=2.69$. The shape stays practically the same after $\xi$ $=1400$, thus confirming its stability. The course of the above transformation is clearly seen in Fig. 7. It is represented by the dotted arrow in Fig. 5. A physically similar process has been considered analytically in [9] for solitons in birefringent fibers. As a general rule, this analysis shows that the transformation always takes place from an upper right point on the $H(Q)$ diagram to a lower left point on the diagram. Hence the direction of the arrow in Fig. 5 must be down and to the left.

The instability eigenvalues of the linearized equations for the upper soliton branch must be complex, as they have real parts which correspond to the deviation from the unstable soliton and imaginary parts which correspond to interactions with radiation. This type of complex eigenvalue has been found for a different problem in [29]. Note that complex eigenvalues have been proved to exist for Hamiltonian systems in [30-32].

\section{CONCLUSION AND DISCUSSION}

We have reformulated soliton stability principles by proving a general theorem for stability in terms of $H$ and $Q$, and we have shown that parametric curves of Hamiltonian versus energy are useful in the theory of solitons in conservative nonintegrable systems. In particular, for lowest-order solitons, concave down implies stability, while concave up corresponds to instability. Furthermore, stability changes only at cusps. We have shown that it is possible to find the Hamiltonian and energy for solitons of non-Kerr-law media without any knowledge of the functional form of the soliton itself. We gave various examples. We also considered some simple dynamics, namely, the transformation of an unstable soliton into a soliton of a stable branch.

We believe that this approach can be generalized to include more complicated Hamiltonian nonlinear systems, including cases with two [8,10] or more coupled NLSEs [33], parametric solitons [34], and examples of higher-order dimensionality. For example, the curves $H(Q)$ calculated numerically in [12] show clearly that our stability criterion can be applied to a system of coupled NLSEs. The results obtained in [14] also show that this principle can be generalized to the case of $(1+3) \mathrm{D}$ solitons. It is quite obvious, then, that $(1+2) \mathrm{D}$ cases and spatiotemporal $(1+3) \mathrm{D}$ solitons [3537] also could be handled with our approach. This means that, independent of their physical nature, single-soliton solutions of Hamiltonian systems can be well understood and analyzed using the concavity of the $H(Q)$ curves.
[1] H. Goldstein, Classical Mechanics, 2nd ed. (Addison-Wesley, New York, 1980).

[2] V. E. Zakharov, Izv. Vyssh. Uchebn. Zaved., Radiofiz. 17, 431 (1974) [Radiophys. Quantum Electron. 17, 328 (1976)].

[3] L. D. Faddeev and L. A. Takhtajan, Hamiltonian Methods in the Theory of Solitons (Springer-Verlag, Berlin, 1987).

[4] V. E. Zakharov and A. M. Rubenchik, Zh. Éksp. Teor. Fiz. 65, 997 (1973) [Sov. Phys. JETP 38, 494 (1974)].

[5] J. J. Rasmussen and K. Ripdal, Phys. Scr. 33, 481 (1986).

[6] N. N. Akhmediev and A. Ankiewicz, Solitons: Nonlinear 
Pulses and Beams (Chapman \& Hall, London, 1997).

[7] N. N. Akhmediev, Opt. Quantum Electron. 30, 535 (1998).

[8] E. A. Ostrovskaya, N. N. Akhmediev, G. I. Stegeman, J. U. Kang, and J. S. Aitchison, J. Opt. Soc. Am. B 14, 880 (1997).

[9] A. V. Buryak and N. N. Akhmediev, Opt. Commun. 110, 287 (1994).

[10] J. M. Soto-Crespo, N. N. Akhmediev, and A. Ankiewicz, J. Opt. Soc. Am. B 12, 1100 (1995).

[11] N. N. Akhmediev, A. V. Buryak, J. M. Soto-Crespo, and D. R. Andersen, J. Opt. Soc. Am. B 12, 434 (1995).

[12] Y. Chen, Phys. Rev. E 57, 3542 (1998).

[13] D. Artigas, L. Torner, and N. N. Akhmediev, Opt. Commun. 143, 322 (1997).

[14] N. N. Akhmediev, M. P. Das, and A. Vagov, in Condensed Matter Theories, edited by J. W. Clark and P. V. Panat (Nova Science, Singapore, 1997), Vol. 12, pp. 17-26.

[15] A. V. Buryak and N. N. Akhmediev, Phys. Rev. E 51, 3572 (1995).

[16] N. N. Akhmediev, Zh. Éksp. Teor. Fiz. 83, 545 (1982) [Sov. Phys. JETP 56, 299 (1982)].

[17] F. V. Kusmartsev, Phys. Rep. 183, 1 (1989).

[18] V. E. Zakharov and V. S. Synakh, Zh. Éksp. Teor. Fiz. 68, 940 (1975) [Sov. Phys. JETP 41, 465 (1976)].

[19] A. A. Kolokolov, Prikl. Mekh. Tekh. Fiz. N3, 152 (1973) [J. Appl. Mech. Tech. Phys. N3, 1426 (1973)].

[20] M. Grillakis, J. Shatah, and W. Strauss, J. Funct. Anal. 74, 160 (1987).

[21] C. K. R. T. Jones and J. V. Moloney, Phys. Lett. A 117, 175 (1986).

[22] D. J. Mitchell and A. W. Snyder, J. Opt. Soc. Am. B 10, 1572 (1993).

[23] D. E. Pelinovsky and R. H. J. Grimshaw, in Nonlinear Insta- bility Analysis, edited by L. Debnath and S. Choudhury, Advances in Fluid Mechanics Series (Computational Mechanics Publications, Southampton, U.K., 1997), Chap. 8, pp. 245312.

[24] I. Bialynicki-Birula and J. Mycielski, Phys. Scr. 20, 539 (1979).

[25] A. W. Snyder and D. J. Mitchell, Opt. Lett. 22, 16 (1997).

[26] S. Gatz and J. Herrmann, Opt. Lett. 17, 484 (1992).

[27] A. E. Kaplan, Phys. Rev. Lett. 55, 1291 (1985).

[28] W. Królikowski, N. Akhmediev, and B. Luther-Davies, Phys. Rev. E 48, 3980 (1993).

[29] I. V. Barashenkov, D. E. Pelinovsky, and E. V. Zemlyanaya, Phys. Rev. Lett. 80, 5117 (1998).

[30] H. T. Tran, J. D. Mitchell, N. N. Akhmediev, and A. Ankiewicz, Opt. Commun. 93, 227 (1992).

[31] I. V. Barashenkov, M. M. Bogdan, and T. Zhanlav, in Nonlinear World, Proceedings of the Fourth International Workshop on Nonlinear and Turbulent Processes in Physics, Kiev, 1989, edited by V. G. Bar'yakhtar et al. (World Scientific, Singapore, 1990), p. 3; I. V. Barashenkov, M. M. Bogdan, and V. E. Korobov, Europhys. Lett., 15, 113 (1991).

[32] N. N. Akhmediev, A. Ankiewicz, and H. T. Tran, J. Opt. Soc. Am. B 10, 230 (1993).

[33] N. N. Akhmediev and A. V. Buryak, J. Opt. Soc. Am. B 11, 804 (1994).

[34] L. Torner, D. Mihalache, M. C. Santos, and N. N. Akhmediev, J. Opt. Soc. Am. B 15, 1476 (1998).

[35] N. N. Akhmediev and J. M. Soto-Crespo, Phys. Rev. A 47, 1358 (1993).

[36] D. E. Edmundson and R. H. Enns, Opt. Lett. 17, 586 (1992).

[37] S. Blair and K. Wagner, Opt. Quantum Electron. 30, 697 (1998). 